\title{
COMMISSION 19: ROTATION OF THE EARTH (ROTATION DE LA TERRE)
}

\author{
Report of Meeting, 24 August 1973
}

PRESIDENT: H. M. Smith.

SECRETARY: H. J. Abraham.

\section{First Session}

The President opened the meeting and called on W. Markowitz to speak on the Independence of the Definitive ILS Polar Motion from Star Catalogues. Markowitz described how Carnera had obtained the coordinates of the Pole separately for each group for each month from the equations $x=\Sigma a_{j} \Delta \varphi_{j} ; y=\Sigma b_{j} \Delta \varphi_{j} ; \Sigma a_{j}=\Sigma b_{j}=0$. Consequently Carnera's definitive declinations and definitive $x$ and $y$ are independent of the initial catalogue. This had been demonstrated by the method of Control Latitudes and by comparison with Fichera's results which are based on the accurate catalogue of Melchior and Dejaiffe. It follows that no change in accuracy in the determination of the periodic or secular motions would result from a change in the conventional latitudes. The CIO should be retained for continuity.

In a further paper describing the ILS and BIH Secular Motions of the Pole for 1962 to 1970 , Markowitz showed that for this period the secular motion from the BIH results, based on numerous stations, was in general agreement with that from the five ILS stations, namely a motion approximately along the $135^{\circ} \mathrm{W}$ meridian. H. J. Abraham added that the rate of increase in the period of the librations that are shown by the ILS data is remarkably constant.

C. A. Murray was asked by the President to present two proposals by R. d'E Atkinson for discussion. The first proposal was that the zero point of right ascension on the true equator of date, used in published ephemerides including the Day Numbers shall be defined by the intersection of the true equator of date with the great circle containing the true pole of date and the mean equinox of date. The second proposal was that the luni-solar nutation used in the ephemerides, including the Day Numbers, shall correspond to that of the Earth's pole of figure and not, as at present, to that of the instantaneous pole of rotation. $T$. Lederle considered that these proposals should be considered in conjunction with others of which notice had been given. He had strong objections to the first proposal. The Chairman suggested that no decisions should be taken at this stage and that the proposals should be fully considered before the next Assembly.

R. O. Vicente was invited by the President to speak about certain observations obtained in Rio de Janeiro. Vicente explained that these had been made from 1924 to 1931 with an instrument that was similar to the ILS instruments and that they were now being reduced. P. J. Melchior offered to obtain corrections for the star positions.

The President then asked S. K. Runcorn to lead a discussion on the Irregular Rotation of the Earth. Runcorn first distinguished the secular changes due to lunar tidal friction, and the irregular changes which could not be ascribed to surface effects. He referred to Vestine's evidence that the Earth's electromagnetic field was drifting by about 0.2 per year, and then discussed the dynamo theory and the consequences of having to conserve momentum. There must have been exchanges of material or changes in the moment of momentum. Urey's suggestion that iron might be finding its way from the mantle to the core could explain a progressive change but the irregular slowing down and speeding up must be due to variations in coupling. It appeared that sufficiently strong currents could occur in the lower mantle as silicates would be semi-conducting at such high temperatures. The torque required to change the length of the day could be calculated. Similar impulsive torques about other axes would cause polar displacements, and such shifts were in fact observed. The polar wobble could arise from shifts of mass, which would displace the mean pole from the axis of figure. He suggested that it could also be caused by impulsive torques that would change the radius of the 
polhode but leave the mean pole unchanged. Examples of this could be seen from Guinot's analysis of the Chandler wobble. There was a possibility of fluctuations being caused through differences in the ellipticities and precessions of the mantle and the core, plus electromagnetic coupling. N. Sekiguchi remarked that the so-called irregular changes were not so irregular. The fluctuations had followed a sawtooth pattern that repeated almost every 30 years, except between 1920 and 1940 , during which period the polar motion had been irregular. He ascribed this to anomalous action of the core. In reply to questions from Markowitz, Runcorn added that energy would be transferred through changes of field. It was important to know whether changes in the length of the day were sudden or gradual. Brouwer had fitted parabolic arcs although this implied sudden changes in torque.

L. V. Morrison was invited by the President to present a paper on Decade Fluctuations in the Rate of Rotation of the Earth. Morrison said that he had used 25000 occultation observations, obtained between 1955.5 and 1972, in fitting the coefficients in the expression for the Moon's mean longitude to the atomic (TAI) time scale. He had then used this expression for the Moon to analyse occultations before 1955.5. He had obtained $-20^{\prime \prime} T^{2}$ where Spencer Jones had used $-11^{\prime \prime} 22 T^{2}$ in expressing the acceleration in the lunar mean longitude. The results then indicated that since about A.D. 1600 the length of the day had fluctuated irregularly with semi-amplitude about $4 \mathrm{~ms}$ and had increased steadily by $1.5 \mathrm{~ms} / \mathrm{cy}$. Ancient data gave $2 \mathrm{~ms} / \mathrm{cy}$ over a time base of 2000 years, but conservation of angular momentum in the Earth-Moon system implied $4 \mathrm{~ms} / \mathrm{cy}$. S. K. Runcorn recalled that Spencer Jones had shown that lunar effects were distinguishable but solar effects could not be isolated from the effects of internal changes. C. A. Murray said in regard to the secular acceleration term that in 1957 he had obtained $-22^{\prime \prime} T^{2}$ from the magnitudes of ancient eclipses, which were independent of position on the Earth's surface and hence were independent of the Earth's rotation. J. D. Mulholland added that compatible values had been obtained by Oesterwinter and Cohen $\left(-19^{\prime \prime} T^{2} \pm 3\right)$ and by Van Flandern. The study by Oesterwinter and Cohen might be regarded as a logical equivalent of the work by Spencer Jones but with the advantages of a more comprehensive internally consistent model and of over a decade of data based on an atomic time scale. The currently available lunar theory was adequate for reliable determinations of the secular acceleration.

B. Guinot was asked by the President to speak on his determination of the Chandler wobble. Guinot explained that he had carefully minimized the effects of changes in the catalogue or the observatories. He had found amplitude changes of 0 "r 1 that occurred in less than 1 year. A change occurred about every 5 years and this strongly supported Runcorn's hypothesis. No correlation with lunar data had been found.

W Markowitz was then invited by the President to speak on Changes in the Speed of Rotation of the Earth. Markowitz referred to Brouwer's use of parabolic arcs and remarked that many other curves could be fitted. He discussed a diagram that showed variations in the length of the day from 1955 to 1970 and noted that Brouwer's and Morrison's data had indicated changes every 10 or 15 years but that recently there had been none. Melchior and Runcorn considered that the choice of parabolae was subjective and presupposed a theory. Winkler remarked that the variations were reminiscent of those in atomic clock comparisons. Markowitz then added that K. Lambeck had found that wind effects caused variations with a period of one to two weeks. Runcorn commented that the suggested change of $G$ in Dirac's hypothesis, and the suggested change in the moment of inertia in Urey's hypothesis, would lead to opposite effects. Markowitz went on to say that the probable errors in the length of day variations were very small. He said also there was no correlation with earthquakes. The Chairman remarked that investigations by O'Hora confirmed Markowitz's views. Winkler asked whether ocean currents could account for the changes but Runcorn replied that there were many orders of magnitude in the uncertainty as to the influence of such currents.

The President then thanked the speakers for their papers and declared the session closed.

\section{Second Session}

The President opened the second session which was devoted to administrative matters. He asked 
the Director of the BIH to present his report. B. Guinot said that, following preliminary studies, the polar motion derived by the Dahlgren Polar Monitoring Service from Doppler measurements on artificial satellites was now included in the current work of the BIH. The DPMS data had reduced the standard deviation of the 5-day values of $x$ and $y$ respectively from $0 \% 011$ to $0 \% 008$ and from $0 " 010$ to $0 " 008$. The solution based on classical data alone would still be published. The use of Doppler measurements from stations not in the DPMS net showed satisfactory results, and a longterm programme was being inaugurated. A study of the stability of the classical instruments had emphasized the quality of PZTs and the ZTL 180 zenith telescopes. The systematic error in the proper motions of the PZT stars relative to the FK4 appeared to be not significant. The precision of the coordinates of the pole and of UT1 computed from the 80 participating observatories (with classical instruments) was improving. The possibility of further improvement was illustrated by the seasonal variation in precision. The BIH had begun to operate a rapid service in May 1971 under contract to JPL, thus giving $x, y$ and UT1-UTC on a weekly basis with a delay of 3 days. During critical periods daily results were provided with 1 day delay. The results generally agreed with the definitive results to within 0.03 for $\mathrm{x}$ and $\mathrm{y}$, and 0.003 for UT1. Winkler referred to differences between the DPMS and the classical data, and explained that the Doppler observations are affected by ionospheric conditions. Constant harmonics were assumed but seasonal effects must occur. Moreover, there are large effects due to earth tides. The President thanked Guinot for his report.

The President then asked the Director of the IPMS to present his report. S. Yumi said that more than 50 stations and observatories co-operate with the IPMS. Few are in the southern hemisphere, however. The preliminary coordinates of the pole are published in the Monthly Notes with a delay of two months, and the detailed results are published annually. Urgent problems stem from star and station errors, the definition of mean latitude, and discrepancies between the coordinates derived from time and latitude data. It was a matter of particular concern that the ILS results for 1949.0 to 1962.0 , which Cecchini had compiled, were still not published. With regard to PZTs, the second at Mizusawa was completed in 1972, there would soon be one at Kitab, one was to be installed at Cagliari, Sardinia, and it was intended to install one at Gaithersburg and another at Ukiah. The Punta-Indio PZT was in operation but co-operation with Mount Stromlo had been temporarily interrupted. There was interest in installing a PZT at Tjililitan near Jakarta. The Wellington astrolabe could be used if support were provided. Brazilian astronomers had plans for an astrolabe at Macapá $\left(\varphi=0^{\circ} 0^{\prime} 0^{\prime \prime}\right)$ to co-operate with Quito, and for a PZT at about $\varphi=-8^{\circ}$. It had not been possible so far to establish co-operation with the Tientsin ZTL 180 or the Shanghai astrolabe and transit instrument. Many observatories were interested in laser ranging, VLBI and Doppler observations, the results of which should be used in studies of the polar motion. Melchior stated that the IUGG had provided funds for Cecchini's results to be published.

The President thanked Yumi for his report and asked him to present the report of the Working Group on the Pole Coordinates, of which he is President. Yumi explained that the purpose of the Working Group was to organize international co-operation and promote agreement. This referred particularly to collecting and reducing the original data to an appropriate frame of reference and to obtain the best coordinates of the pole. The Working Group favoured the forwarding of all ILS data to Mizusawa, conversion to machine-readable form, and the use of the Melchior catalogue and of constants referred to the present IAU system. It had agreed also on the methods of obtaining the apparent places of the stars and the coordinates of the Sun. Data to be punched would cover 70 years and require about 2 million cards. On behalf of the Commission the President thanked Yumi for this report.

It was agreed by the meeting that the names of the following members should be submitted to the Executive Committee for future office.

President: C. Sugawa

Vice President: R. O. Vicente

Organizing Committee: H. J. Abraham, P. L. Bender, M. H. Cohen, K. Lambeck, V. Milovanovic, P. Pâquet, H. M. Smith, Ya. S. Yatskiv, S. Yumi (ex officio). 
Resolutions recognizing the importance of VLBI and laser ranging to the Moon and artificial satellites were moved by Markowitz and seconded by Melchior. They were strongly supported by Bender and were passed unanimously.

In conclusion the President expressed his appreciation of the work by members of the Commission, and a vote of thanks to the President was carried with acclamation.

Resolution Adopted by Commission 19:

whereas new methods have been developed for determining the angular position of the Earth by long baseline radio interferometry and by laser range measurements to the Moon and to artificial satellites; and

whereas the accuracy expected from these techniques for determining polar motion, UT1, and crustal movements is very high; and

whereas more accurate determination of these quantities is expected to give valuable information on earthquake mechanisms, on the transfer of angular momentum between the atmosphere and the Earth, and on interaction between the core and the mantle; and

whereas the importance of some of this information is great for practical applications as well as for scientific purposes;

it is therefore recommended that: preliminary multi-national and global networks of stations to determine polar motion and Earth rotation by these techniques be established; and

that these global networks be operated for a long enough time to permit both an evaluation of the accuracies achievable by these techniques and a comparison of the results with those obtained by other methods; and

that additional technical developments be undertaken to improve further the accuracy of these methods.

\section{Joint Session of Commission 19 and 31, 24 August 1973}

ChaIrman: H. M. Smith.

SeCReTARY: H. Enslin.

The Chairnan opened the session devoted to new developments of conventional instruments for the determination of UT and Polar Motion.

S. Yumi described the new PZT at Mizusawa which has been in operation since May 1972. It has been installed in an underground room; temperature and humidity are kept constant. The plate carriage is driven by a stepping motor; photo-electric timing is employed. A cylindrical dew-cap goes up from the lens into the open air. A problem still to be solved arises from air mass movements in the cylinder in winter when the outside temperature is below that of the instrument.

The chairman noted that the Herstmonceux PZT has also been equipped with a stepping motor. The accuracy achieved in the time coordinate is inferior to that obtained in the latitude coordinate: a moire fringe timing unit is being fitted in an endeavour to improve the timing accuracy.

G. Winkler reported on the new $65 \mathrm{~cm} / 13 \mathrm{~m}$ PZT of the U.S. Naval Observatory. Special features are: four-element apochromatic lens, field of view of about one square degree, variable length shutter timing mechanism, facility for automatic plate changing, pneumatic device for rotating the head. Great care will be taken to avoid thermal disturbances, especially by keeping the instrument during the day below the expected night temperature. All heat-producing equipment will be removed some hundred feet from the instrument. It will be operated by an IBM Data Acquisition System and stars down to $11 \mathrm{~m}$ will be observed. The lens is corrected for $5000-6500 \AA$ with a filter for cut off at $5000 \AA$. Tidal variations of the plumb line will be checked by a mercury tiltmeter. At some later date, photo-electric observation might be considered: it promises no higher accuracy than the use of photographic plates, but it would eliminate the plate measuring.

Discussion arose on the problem of tiltmeter drift, following remarks by S. Iijima. All speakers agreed on the importance of geological and underground features. Winkler said that the tiltmeter at the U.S. Naval Observatory drifts in rain because it has been installed on a slope. He pointed 
out that by cross-correlation of tiltmeter and astronomical observations the tiltmeter drift can be determined. $\mathbf{J}$. Terrien referred to new ideas on measuring mercury levels. The chairman said that the use of a tiltmeter is being considered at the Royal Greenwich Observatory.

$S$. Débarbat outlined the photo-electric astrolabe being studied at the Paris Observatory by $G$. Billaud. It is basically similar in construction to the full aperture astrolabe APP but a mirror will be used instead of the lens. For photo-electric observing, a fixed grid is used in conjunction with a photomultiplier tube which collects the modulated light from a star image passing the successive slits of the grid (photon-counting technique according to Høg's principle is employed). It will probably be possible to observe all FK4 stars and a reduction of errors is expected from impersonal observations as well as from several instrumental improvements. By changing the optical square, observations can be made at different zenith distances, e.g. for deducing absolute declinations.

Technical details of the astrolabe were discussed by Guinot and others. Winkler mentioned that experiments with automatic registration have been made by $D$. Monger with one of the two Richmond astrolabes. Two methods have been tested. A photo-electric camera has been used in one case, a photographic technique in the other case.

C. Costain proposed that the work of the PZT's and astrolabes which form chains should be encouraged. A resolution was drafted by W. Markowitz and adopted by the Commission. (Resolution attached.)

The Chairman closed the session.

\section{Joint Resolution by Commissions 19 and 31}

\section{Considering that}

Optical observations made with chains of PZT's and astrolabes which observe the same stars provide basic data for studies of the secular motion of the pole and continental drift, and that the establishment of such chains has been recommedend by the IAU,

Resolution

Urges that such existing chains, for example, Calgary - Herstmonceux and Punta-Indio Mt. Stromlo, shall be fully supported by the administrative agencies concerned.

\section{Proposal to the Executive Committee for a Motion by Commissions 4, 19 and 31}

Commissions 4, 19 and 31 invite attention to the increasing work load of the $\mathrm{BIH}$ for the international coordination of time of high precision, which is needed now for satellite and deep space tracking and for radio astronomy (e.g. pulsars, VLBI, lunar laser ranging and precise coordinates). The BIH is the only agency which provides this important service for astronomy (as well as for other sciences).

Therefore, Commissions 4,19 and 31 urgently request that a subsidy of $\$ 12000$ per year be granted to the BIH.

\section{August 1973}

\section{NEW TECHNIQUES FOR DETERMINATION OF UT1 AND PV}

Chairman: H. M. Smith.

SECRETARY: L. V. Morrison.

C. Counselman (MIT) spoke on the results obtained from wide-band Very Long Base Interferometry. The technique consisted of measuring the time delay between widely spaced antennae of wave-fronts from extragalactic sources whose relative positions were known with an accuracy of about 0.01 . These data together with an atomic clock pulse were recorded on magnetic tape at each antenna and later analysed using the clock pulses to establish the coherence of the signals. The difference in clock rates was eliminated by observing four different sources in each set of observa- 
tions. The present accuracy of the measured time delay, expressed in angular resolution, was of the order of $0 \% 05$, but it was likely that this would be improved by a factor of 10 . A comparison of four results for the $x$-component of polar motion agreed with the BIH values to about 2 metres, and the UT1 results agreed with the USNO PZT values to about $5 \mathrm{~ms}$. It was found that the N-S base line varied by about 2 metres. P. Bender asked if the comparisons had been made with the mean BIH 5-day values. B. Guinot advised using the definitive values in the BIH Annual Reports. N. Matsunami enquired about the stability of the masers, and Counselman said that clock jumps could be easily identified. In reply to a question by L. Morrison, Counselman stated that 17 sources were used, but that there are more with sufficiently accurate positions. I. Soltau asked if Earth tides could explain the 2 metre variation: P. Melchior said that they are no more than $\frac{1}{2}$ metre.

B. Elsmore (Mullard Radio Astronomy Observatory, Cambridge, U.K.) reported on results obtained using a linked E-W interferometer comprising two aerials separated by $2 \mathrm{~km}$. A radio source of known position was tracked continuously from $-6^{\mathrm{h}}$ to $+6^{\mathrm{h}}$ in hour angle. The measured phase difference gave UT1 directly, as the measurements were independent of the instantaneous position of the pole. The accuracy of UT1 realized from one passage of a radio source was $\pm 4.6 \mathrm{~ms}$, which is to be compared with the value of $\pm 4.0 \mathrm{~ms}$ for a single night's observation using a PZT. A limitation of the method is the irregularity of water vapour in the troposphere. B. Guinot regarded an accuracy of $\pm 4.0 \mathrm{~ms}$ as too optimistic for the results of one night using a PZT

C. Oesterwinter (U.S. Naval Weapons Laboratory) reported on the results of polar motion for 1972 obtained by R. Anderle using doppler observations of artificial satellites. The standard error for the determination of the position of the pole based on 5 days of observations was as low as $20 \mathrm{~cm}$ during periods when data from 19 stations on 3 satellites were used. The $x$ and $y$ components of polar motion were in good agreement with the BIH values. P. Melchior (Royal Observatory, Belgium) reported on the work of P. Pâquet and R. Dejaiffe who had intercompared the results from doppler and astrolabe observations, both made at the Royal Observatory. They had carried out an analysis deducing the internal and external standard deviations due to both methods. In order to reduce the standard deviation in longitude for the doppler results to that of the astrolabe, about $5 \%$ of the satellite passes had to be rejected. A comparison of the doppler latitude results with BIH values for 1972 showed a small systematic shift in phase and amplitude. B. Guinot remarked that the phase-amplitude difference was not surprising; the results were subject to annual fluctuations. $\mathbf{P}$. Melchior agreed and suggested that in the case of doppler measurements, annual variation in the troposphere should be investigated. W. Markowitz asked if the results quoted for the coordinates were astronomical or geodetic. Melchior said that as derived from the doppler measurements, they were geodetic, using the NWL Earth model. J. Mulholland agreed that there are reasons for transforming station coordinates determined geocentrically into some geodetic system, but that for global work the geocentric values are the most useful. He asked that these also be published. Bender accepted the drift in excess of $50 \mathrm{~cm} / \mathrm{yr}$ earlier reported by Oesterwinter as possible for the Japanese and Philippine stations, but not for the U.S. ones. He suggested that they might be due to short term phenomena, possibly originating in the ionosphere.

K. Johnston (U.S. Naval Research Laboratory) reported on the determination of UT1 using VLBI measurements of molecular $\mathrm{H}_{2} \mathrm{O}$ sources at $22.2356 \mathrm{~Hz}$. Since the sources have very narrow intrinsic bandwidths, only the fringe rate was measured. For the strongest sources, the fringe rate residuals were about $2.5 \mathrm{mHz}$, and using a $4000 \mathrm{~km} \mathrm{E}-\mathrm{W}$ base line it would be possible to determine UT1 with an accuracy of about $2 \mathrm{~ms}$. G. Winkler asked how the maser clocks were synchronized and suggested that some external means was required because they drift with time. Johnston replied that they were simply measuring a difference in transit time.

P. Bender (National Bureau of Standards and University of Colorado) reported on the use of Lunar Laser Ranging (LLR) to determine UT1 and PV. The 5-day mean BIH values were accurate to about $\pm 40 \mathrm{~cm}\left( \pm 0^{z} 03\right)$ for PV: with about 6 stations distributed in both hemispheres observing at hour angles $-3^{\mathrm{h}}, 0^{\mathrm{h}}$ and $+3^{\mathrm{h}}$ LLR could attain an accuracy of a few centimetres for PV after the removal of lunar ephemeris errors. From a 9- to 10- hour observing period on a particular day the residual from the McDonald observations had a RMS of about $11 \mathrm{~cm}$ which demonstrated 
that there were no short term unknowns in the lunar ephemeris. In future it was hoped that LLR stations would be set up in other countries, particularly in the southern hemisphere, which would undertake programs consisting of range measurements three times daily to at least one reflector in order to detect changes in the Earth's rotation which occur at intervals down to one day. Chairman: Before leaving the meeting, I would like to say that there seems to be a need to coordinate the results on UT1 and PV coming from the various techniques that we have heard about this afternoon. I now invite Professor Melchior to take the Chair. P. Brosche enquired if the solid Earth tides of the order of $20 \mathrm{~cm}$ would affect the measurements. Melchior pointed out that only the radial component was involved; and that this is dependent on the Love number $h$. Bender said that the effect was less than $1 \mathrm{~cm}$. H. Eichhorn referred to the distance of the Moon being measured to about $15 \mathrm{~cm}$, but wondered if the analytical theory was equally accurate. Mulholland said it was over a short period and added that the limitation comes in the angular position of the Moon in the sky and here the accuracy of the ephemerides is at present comparable to the ILE, that is, about 0"75. This was achieved by the synthetic fit of a numerical integration to the ILE.

L. Morrison said that by using occultations this could be improved to $0: 4$. Bender drew attention to the limitation of the libration theory but said that with the extension to include third and fourthdegree terms in the lunar gravity field, fitting of data with a residual RMS of about $2 \mathrm{~m}$ has been achieved: the inclination and node corrections are required. In reply to a further question by Morrison, Mulholland said that no estimates had been obtained for the corrections to the inclination and node, but the problem was being investigated with the co-operation of the USNO.

D. Smith (Goddard Space Flight Center) reported on results obtained from doppler ranging to artificial satellites. After adjustment for the harmonics of the Earth's field, a residual fit with RMS of $50 \mathrm{~cm}$ had been achieved for single passes over 2 to 3 hours. This had later been improved to $30 \mathrm{~cm}$. Tidal deformation had been removed by taking $k_{2}$ as 0.25 . The variation in latitude had been compared with BIH data and the residual RMS was of the order of 0.03. There were plans to launch a high-altitude laser reflector and here the limitation would be due to variations in the Earth's albedo pressure. The aim was to have a daily measure of PV to within a few centimetres and UT1 to $100 \mu \mathrm{s}$.

There then followed a general discussion on the possibility of future use of the new techniques in determining PV and UT1 on a regular and routine basis. In particular, it was considered doubtful whether VLBI could provide a routine service as the technique relied on the co-operation of the existing network of NASA stations and these were at present subject to financial constraints.

The Chairman then closed the session. 